\title{
MicroRNAs as New Bioactive Components in Medicinal Plants
}

Authors

Affiliation
Wenyan Xie, Alexander Weng, Matthias F. Melzig

Institute of Pharmacy, Freie Universität Berlin, Berlin, Germany
Key words

- miRNAs

- medicinal plants

- cross-kingdom

- oral delivery

- therapeutic agent

- bioactive component received March 5, 2016

revised April 22, 2016

accepted April 30, 2016

Bibliography

DOI http://dx.doi.org/

10.1055/s-0042-108450

Published online June 7, 2016

Planta Med 2016; 82:

1153-1162 @ Georg Thieme

Verlag KG Stuttgart · New York .

ISSN 0032-0943

Correspondence

Dr. Wenyan Xie

Institute of Pharmacy

Freie Universität Berlin

Königin-Luise-Str. $2+4$

14159 Berlin

Germany

Phone: + 493083853722

Fax: + 4930838451461

wenyan.xie@fu-berlin.de

\section{Abstract \\ $\nabla$}

Herbal medicine has been used to treat diseases for centuries; however, the biological active components and the mechanistic understanding of actions of plant-derived drugs are permanently discussed. MicroRNAs are a class of small, noncoding RNAs that play crucial roles as regulators of gene expression. In recent years, an increasing number of reports showed that microRNAs not only execute biological functions within their original system, they can also be transmited from one species to another, inducing a posttranscriptional repression of protein synthesis in the recipient. This cross-kingdom regulation of microRNAs provides thrilling clues that small RNAs from medicinal plants might act as new bioactive components, interacting with the mammalian system.

\section{Introduction}

$\nabla$

Endogenous microRNAs (miRNAs) are a class of single-stranded non-coding RNA molecules of approximately 22 nucleotides that play crucial roles in gene expression [1]. They generally bind to complimentary sequences in the 3 ' untranslated region (UTR) of specific protein-coding genes, but can also interact with other regions of the gene including 5' UTR and the coding region, inducing mRNA cleavage or translational repression [2]. miRNAs are highly pleiotropic and a single miRNA can recognize hundreds of mRNA transcripts, allowing them to regulate a diverse range of biological pathways. In mammals, an estimated $60 \%$ of all protein-coding genes may contain miRNA binding sites $[3,4]$. Furthermore, miRNA dysregulation is frequently associated with human diseases such as cancer, cardiovascular diseases, central nervous system diseases, and metabolic disorders [5-8], suggesting their potential as targets for the development of novel therapies for
In this article, we provide an overview of the cross-kingdom communication of plant-derived microRNAs. We summarize the microRNAs identified in medicinal plants, their potential targets in mammals, and discuss several recent studies concerning the therapeutic applications of plantbased microRNAs. Health regulations of herbal microRNAs in mammals are a new concept. Continuing efforts in this area will broaden our understanding of biological actions of herbal remedies, and will open the way for the development of new approaches to prevent or treat human diseases.

Supporting information available online at http://www.thieme-connect.de/products

several diseases. There are currently many efforts focusing on the development of miRNA therapeutics for the treatment of human diseases. Several preclinical studies on therapeutic miRNA replacement have been initiated [9-11], and the first liposomal nanoparticle formulation of miR-34a mimic (MRX34) has entered clinical trials in patients with primary liver cancer [12], highlighting the therapeutic potential of miRNA-based novel approaches to treat diseases. However, severe on-target or off-target side effects could be caused by artificial miRNAs $[13,14]$.

Herbal medicine has been used to treat diseases for centuries. Bioactive secondary metabolites such as polyphenols, alkaloids, saponins, and tannins have been systematically studied and used as an important source for drugs. However, synthetic supplements of phytochemicals often do not have the same efficacy as complex herb materials. The natural biological active components and mechanistic understanding of actions of plant-derived drugs are permanently discussed. 
Herbal remedies may produce a beneficial effect by regulating mammalian miRNA expressions. Medicinal plant extracts and secondary metabolites such as Dioscorea opposita Thunb. (Dioscoreaceae) extract, ginsenoside Rh2, berberine, and waltonitone were reported to modulate disease-associated miRNA levels [1518]. Notably, oral consumption of a plant-based diet may lead to the cross-kingdom transfer of plant-derived miRNAs in mammals. Zhang et al. [19] reported miRNAs derived from plantbased foods function as active signalling molecules to regulate mammalian genes. The authors showed that exogenous miRNAs (e.g., MIR168a and MIR156a) from rice were absorbed by cells of the gastrointestinal (GI) tract of the consuming animal, packaged into exosomes, then migrated through the plasma, and were delivered to specific organs where they bound to low-density lipoprotein receptor adapter protein 1 (LDLRAP1) mRNA and inhibited LDLRAP1 expression, thereby influencing cholesterol transport [19]. These studies provide thrilling clues that miRNAs might act as new bioactive compounds in medicinal plants. If this hypothesis can be validated, it will broaden our understanding of herbal remedies and, more importantly, will provide new insights into the development of miRNA-based therapeutics with high efficiency, reduced side effects, and low costs. This review provides current knowledge of cross-kingdom communication of plant-derived miRNAs, summarizes the miRNAs identified in medicinal plants, and discusses several recent studies concerning the therapeutic applications of medicinal plant-based miRNAs.

\section{Evidence for the Uptake of Plant miRNAs by Mammals $\nabla$}

Immediately after Zhang's group discovery, numerous details of this study have been appraised. In general, follow-up studies have focused on detecting exogenous small RNAs in mammals after different diets. The results remain controversial, as summarized in Table 1, with some findings being consistent with the original publication [19-27] while others are questioning the results [28-32].

Recent reports from two groups have attracted great attention. Yang et al. [20] identified the influence of diet preference and health issues on the uptake of plant-based small RNAs. A significant increase of plant miRNA MIR2911 in both blood and urine was observed in mice that were fed with an herbal diet (Lonicera japonica Thunb., Caprifoliaceae; common name: honeysuckle) for several days. Damaged guts resulting from chemotherapeutic (cisplatin) treatment also enhanced plant-based miRNA uptake. In addition, a single large oral dose of exogenous miRNAs (e.g., $400 \mathrm{pmol}$ of synthetic MIR2911 or MIR168 a) facilitated only the short-term detection of serum miRNAs. The following study demonstrated that the level of circulating MIR2911 was associated with the dietary intake level [33]. Zhang's group raised the critical issues for the accurate measurement of exogenous miRNAs such as RNA extraction method and internal controls [21]. These studies supply reasonable explanations on the publications reporting undetectable exogenous miRNAs. Firstly, small dosages over short time feedings of a plant diet may not induce detectable miRNAs in plasma. Secondly, natural ingredients in an herbal diet may protect plant miRNAs against degradation and enhance the uptake of miRNAs in the mammalian system, while naked miRNAs may not survive the digestion and circulation process. Thirdly, the health conditions of the GI tract may influence the uptake of plant-based miRNAs. Fourthly, not only the plant miRNAs in plasma, but the potential deposition of dietary
miRNAs in other body tissues should also be taken into account. Finally, canonical methods for exogenous plant miRNA measurement are necessary to ensure the sensitivity and precision of miRNA detection.

\section{Plant-Based miRNAs for Therapeutics $\nabla$}

Although the role of exogenous miRNAs is currently not well understood, the biological effects of plant miRNA in the mammalian system have been profiled. Using computational tools, $12 \mathrm{pu}-$ tative miRNAs were identified from Curcuma longa L. (Zingiberaceae) and found to bind with various target genes related to human diseases such as diabetes mellitus type II, cardiovascular disorders, Alzheimer's, cancer, and thalassemia [34]. Similarly, a total of six putative miRNAs were identified from Gmelina arborea Roxb. (Lamiaceae), while the mammalian target genes associated with cancer, blood borne disease, and other urinary infections were predicted [35].

Exosomes are one of the natural carriers of miRNA, serving as mediators in extracellular communication [36]. Using grape exosome-like nanoparticles (GELNs) as models, the biological impacts of plant exosomes have been investigated. GELNs can penetrate through the intestinal barrier, can be taken up by intestinal stem cells, and mediate intestinal tissue remodelling [37]. Oral administration of GELNs protected mice against dextran sulfate sodium-induced colitis. Apart from proteins and lipids, miRNAs, with almost 100 different kinds, were identified from GELNs [37]. Furthermore, exosome-like nanoparticles derived from edible plants, including grapes, grapefruit, ginger, and carrots, were taken up by mouse intestinal macrophages and stem cells. They were biologically active in maintaining intestinal homeostasis through activation of nuclear factor (erythroid-derived 2)-like-2 factor (Nrf-2), induction of anti-inflammatory molecules, and activation of Wnt signalling in recipient cells [38]. These studies suggested that plant miRNAs might be packaged into exosomelike nanoparticles that would be taken up by the intestine, and exert beneficial effects under both physiological and pathological conditions.

The most direct evidence of therapeutic effects of plant miRNAs has been recently published by Zhang's group [22]. A plantderived miRNA, MIR2911, is highly stable in the decoction of a Chinese herb honeysuckle, which has been traditionally used in China for centuries to treat influenza infection. This miRNA can be absorbed by the GI tract and be delivered via the bloodstream to the lungs of the animals, which were continuously fed with a honeysuckle decoction. Importantly, this plant miRNA could directly target various influenza A viruses, suppress their replication process, and protect against influenza virus infections in mice. The region of the influenza genome that MIR2911 targeted was also identified, and validated in a mutant virus in which the sequence was modified and resistant to MIR2911 treatment [22]. This groundbreaking study suggested that plant miRNA may act as a novel bioactive ingredient that can be absorbed by mammals and reduce the risk of many diseases.

Recently, the therapeutic potential of plant-based miRNAs in cancer was experimentally confirmed [23]. A cocktail of three mammalian tumor suppressor miRNAs was synthesized with methyl groups on the 2 ' position of the ribose of the 3 ' nucleotide to mimic miRNAs of plant origin. Oral administration of these plant-based tumor suppressor miRNAs together with total plant RNA [isolated from Arabidopsis thaliana (L.) Heynh (Brassica- 
ปัّ

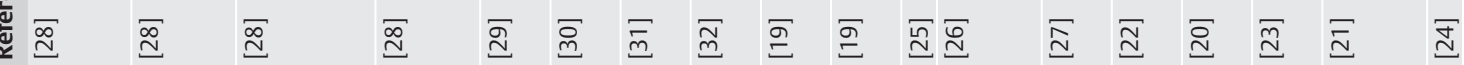

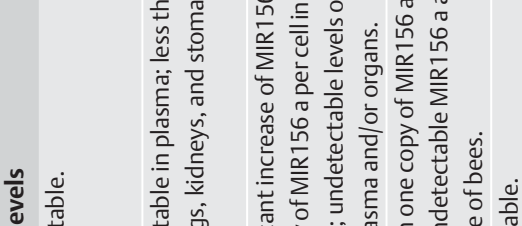

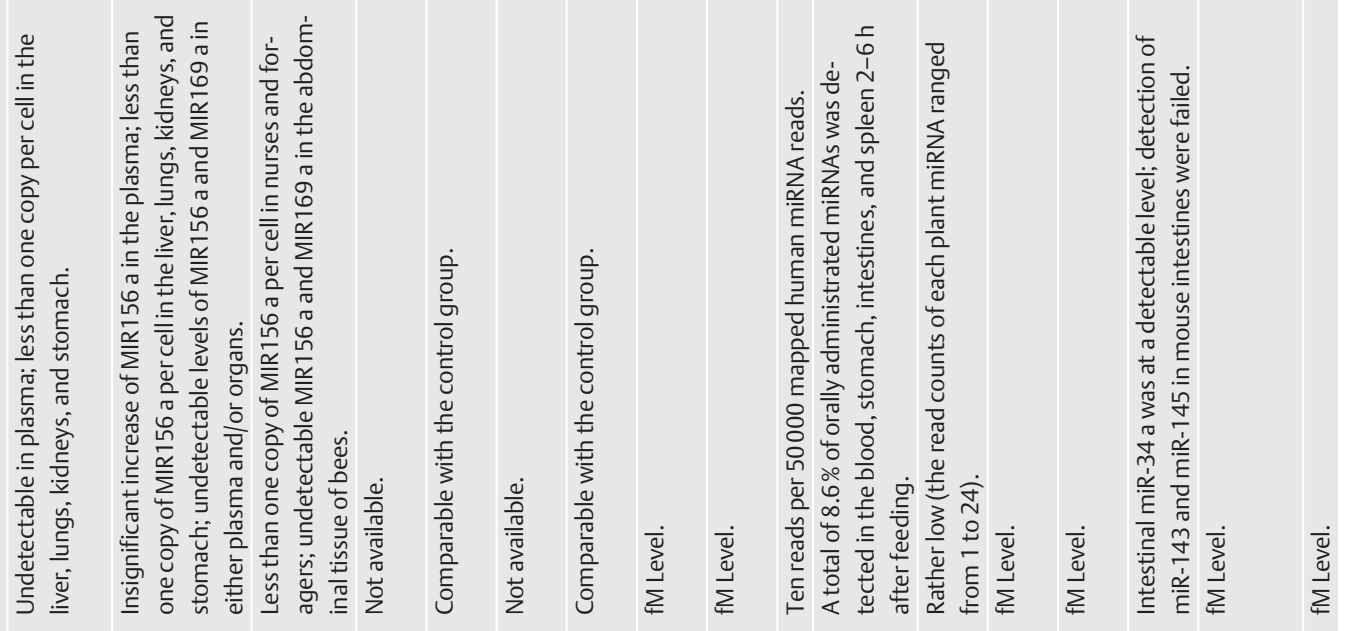

늠

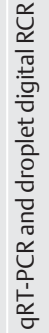

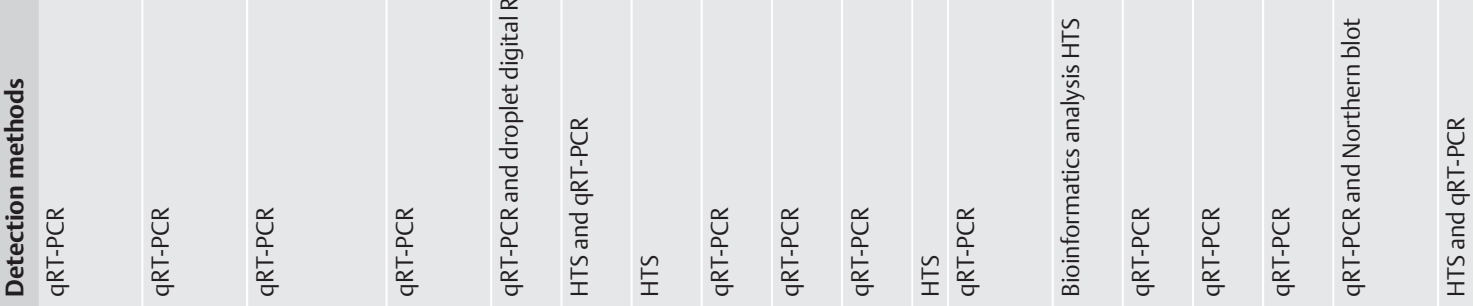

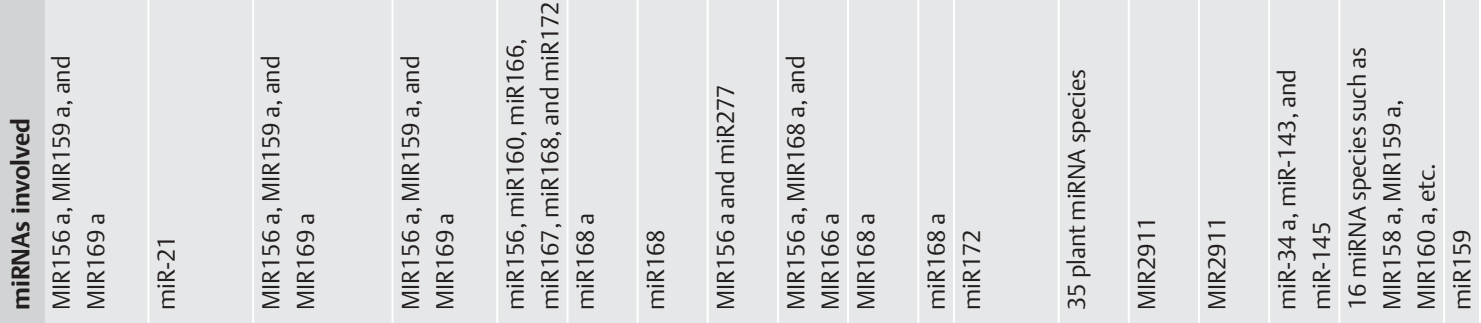

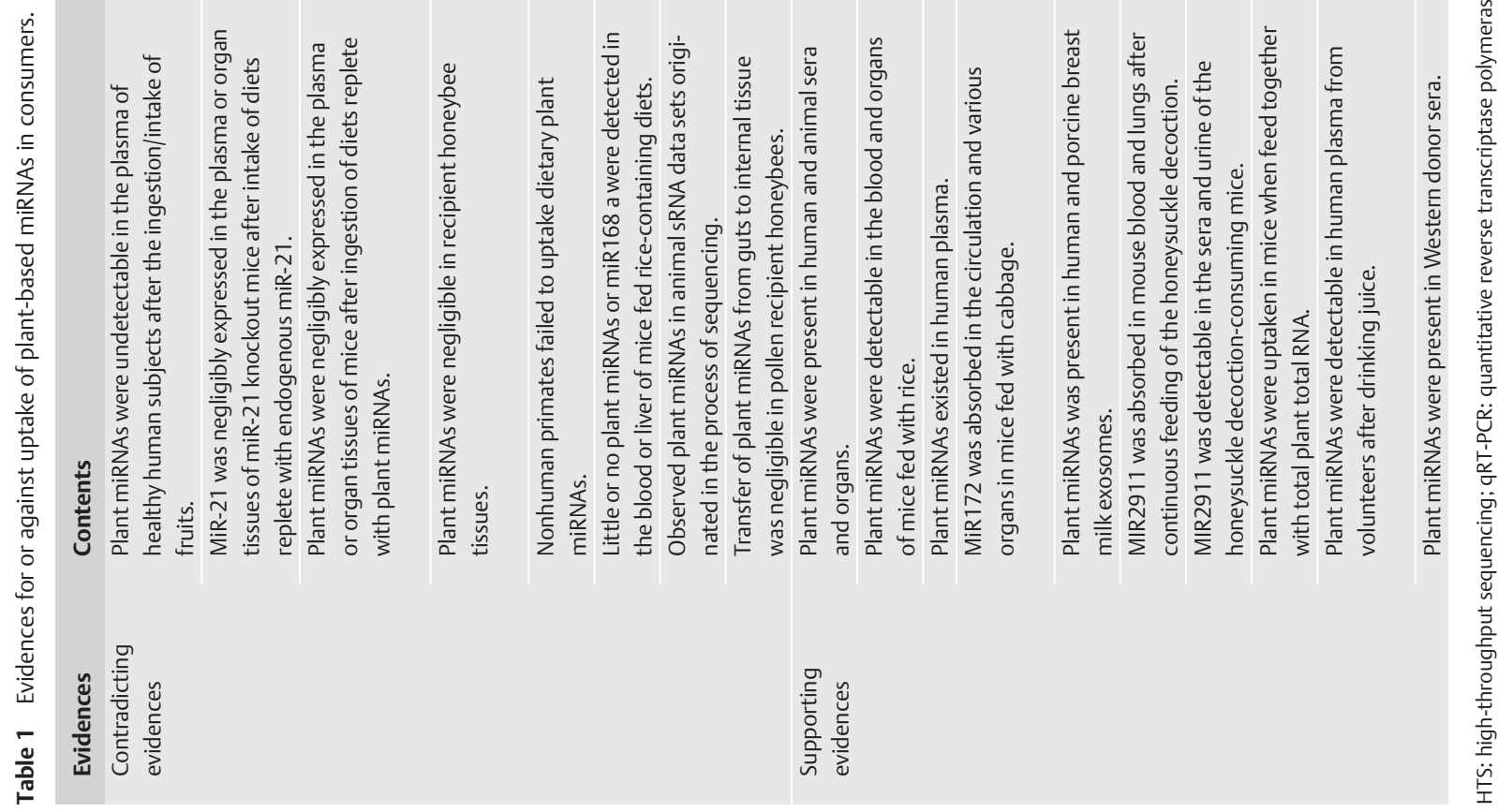


ceae)] significantly reduced the tumor burden in a well-established $A p c^{\mathrm{min} /+}$ mouse model for colon cancer. Interestingly, there was a slight tumor burden reduction in the mice treated with total plant RNA alone compared with water-treated mice, although not statistically different, suggesting that plant total RNA may have a therapeutic effect [23]. This study indicated that plant engineered miRNAs that are designed for specific targets have significant potential in clinical therapeutic applications.

The cross-kingdom regulation of human cancer growth by plant miRNA has been further confirmed using in vitro and in vivo models [24]. Plant-derived miR159, presented in both raw and cooked foods (e.g., broccoli), was not only abundant in healthy human serum, but also detectable in breast cancer tissues. By targeting 3'UTR of transcription factor 7 (TCF7), both synthetic 2-Omethylated miR159 and human serum extracellular vesicles (EVs) significantly suppressed breast cancer cell proliferation. In tumor xenograft models, the mice that were continuously fed with synthetic miR159 showed significantly reduced tumor growth compared with those that received scrambled control oligonucleotides. However, no affection was observed in the xenograft tumors that stably overexpressed TCF7 without the 3'UTR [24]. This study consolidated the assumption that circulating miRNAs absorbed from dietary plants can be transported through EVs-mediated trafficking, reach target tissue, and impact human health and disease.

It is noteworthy that reaching biologically active levels of circulating plant miRNAs through oral intake might require feeding with an herbal decoction, ground plant material, or food matrix $[19,22,37]$ together, or long-term feeding regimes [22-24], suggesting that a plant-derived matrix as well as prolonged exposure might be the important factors that facilitate the high level uptake of plant miRNAs with therapeutic effects.

\section{Stability of Plant miRNAs}

$\nabla$

A crucial concern regarding therapeutic plant miRNAs is their stability in the preparation process as well as in mammalian circulation. The innate capacity of plant-derived miRNAs (e.g., miR166, miR167, miR168, and miR159) are degradation resistant, as evidenced that storage, processing, and cooking do not abolish the plant miRNAs present in rice, soybeans, potatos, broccoli, and cabbage $[19,24,39]$. Unlike mRNA that losses integrity during processing, plant miRNAs are robust during soaking, boiling, and homogenization processes, and even survive under unfavorable physiological conditions such as an extreme $\mathrm{pH}$ (e.g., simulated gastric fluid with $\mathrm{pH}$ of 1.2), frequent bowel movements, and potent ribonuclease (RNase) treatment $[19,39]$. However, it is also reported that the majority of miRNAs were degraded in the honeysuckle decoction after boiling for $30 \mathrm{~min}$, while MIR2911 possesses a specific sequence and high GC content, which makes it highly stable in this decoction and resistant to RNase treatment [22]. These observations suggested that some plant-based miRNAs are in a stable form that can withstand not only the preparation process, but also the digestion and circulation process, ensuring their regulatory activity.

Several mechanisms regarding the stability of plant miRNAs have been suggested:

1. 2'-O-Methylation in plant miRNAs enhances their stability by protecting them from exonucleolytic digestion and uridylation $[40,41]$;
2. Stabilization of circulating miRNAs is associated with RNAbinding proteins such as Argonaute proteins (AGOs) [42-44] and nucleophosmin 1 [45];

3. miRNA carriers such as exosomes, microvesicles, and highdensity lipoprotein protect miRNAs from degradation $[38,46$, 47];

4. The unique sequence and GC content of a plant miRNA also influences its stability [22];

5. Some plant extracts and secondary metabolitess such as extracts from Phyllanthus reticulatus Poir. (Phyllanthaceae), Ananas comosus (L.) Merr. (Bromeliaceae), Aglaia aphanamixis Pellegr. (Meliaceae), green tea, and polyphenols (e.g., epigallocatechin gallate, epicatechin, epicatechin gallate, and epigallocatechin) possess potent ribonuclease inhibitory activities [4851], which may protect plant miRNAs from the enzymatic environment of the digestive tract;

6. The lipid, proteins, or polysaccharides presented in plants may protect miRNAs or miRNA carriers from degradation during preparation and processing.

\section{Oral Delivery of Plant miRNAs \\ $\nabla$}

Oral application is the dominant route of application for herbal preparations. Even if they are stable during preparation and digestion processes, could these exogenous miRNAs cross biological barriers? Despite increasing efforts, the mechanisms of functional transfer of plant miRNAs to cross the intestinal barrier remain unclear. Given the instability of naked RNA, it would be possible that miRNAs in plant materials are packaged into protein complexes and/or lipid vesicles and recognized by a mammalian transport system. Several mechanisms for the intestinal permeability of exogenous miRNAs have been proposed:

1. The RNA-containing complexes are internalized into the intestinal epithelia and become engulfed exosomes. Some of them may merge with endosomes and undergo transcytosis that transports macromolecules from one side to the other side of a biological barrier, while some other fused endosomes mature into lysosomes and undergo degradation $[52,53]$.

2. The RNA carriers might be transferred through $M$ cells in Peyer's patches of the intestine to macrophages or/and dendritic cells in the gut-associated lymphoid tissue (GALT). These GALT immune cells contribute to the subsequent distribution of RNA-containing complexes throughout the body [54,55].

3. Plant miRNAs packaged within exosome-like nanoparticles could be taken up by intestinal stem cells or macrophages where they may exert biological functions $[37,38]$.

4. Although naked plant miRNAs are instable and may survive only for a few hours [19], it is possible that these unprotected miRNAs are uptaken into the cytoplasm by receptor-mediated endocytosis or transmembrane miRNA transporters [52-56].

5. Additionally, altered intestinal permeability resulting from GI tract diseases, stress, malnutrition as well as folk remedies may enhance the uptake of foreign miRNAs $[23,55]$.

Considering that the uptake of plant miRNAs in mammals is not necessarily related to the abundance of miRNAs in the plants [19, 24 ], there might exist a class of plant miRNAs or miRNA carriers with specific signatures that are recognized and preferentially taken up by the mammalian GI tract. After crossing the intestinal barrier, plant miRNAs or miRNA carriers might be repacked into microvesicles and delivered to different tissues/cells where the miRNAs modulate the expression of their target genes [19,22]. 
In mammalian organisms, proteins at the surface of miRNA-containing lipid vesicles take part in the target-specific internalization into cells, such as exosome surface proteins tetraspanins and lectins, while integrins, proteoglycans, and lectin receptor proteins on the surface of recipient cells may serve as receptors for the exosome uptake [57]. However, the molecules on the plant miRNA carriers responsible for the interaction with the mammalian GI tract as well as the target cells have not been elucidated.

It is necessary to mention that not all the ingested miRNAs end up in the bloodstream with potential functions. The level of exogenous miRNA matters to their biological function. It is generally considered that a threshold concentration of 100 copies per cell is required for miRNAs to execute their function [22]. From the published data, although some plant-derived miRNAs (e.g., miR160 a, miR162 a, and miR390 a) are detected in human serum [19], they may not be biologically active.

\section{miRNAs in Medicinal Plants}

$\nabla$

Identification of plant miRNAs is the prerequisite for the understanding of their functions. Genetic and computational approaches are usually used for the investigation of miRNAs. Based on the publically available genome sequence or expressed sequence tags (ESTs), computational methods have been used for the prediction of evolutionary conserved plant miRNAs among different species. However, except for intensively studied herbs such as Panax ginseng C.A.Mey (Araliaceae), Salvia miltiorrhiza Bunge (Lamiaceae), Lycium chinense Mill. (Solanaceae), and Cynara cardunculus L. (Asteraceae) [58-61], limited genomic data are available for medicinal plants. With the recent advent of highthroughput sequencing (HTS) technology, characterization of miRNAs through sRNA, transcriptome, or genome sequencing has become a routine tool for miRNA research.

According to our current knowledge, a total of 44 medicinal plants with identified miRNAs are available in PubMed, Science Scope, and miRase databases ( 0 Table 2 ). With the aid of computational approaches, the functions of plant miRNA have been predicted. They are involved in various plant biological processes, including plant growth development, signal transduction, and stress responses [62-66]. Secondary metabolites have generally been considered as the pharmacological active compounds in medicinal plants, and the role of miRNAs in regulating the production of bioactive secondary metabolites has gained high attention [67-71].

It is noteworthy that even the efficacy of some herb remedies [e.g., Viscum album L. (Santalaceae) or Vitex agnus-castus L. (Lamiaceae) has been clinically demonstrated, though the scientific explanation of their pharmacological activities are not convincing from the experimental data about concentration, pharmacological activity, bioavailability, and metabolism of secondary metabolites present in the used extracts. One possibility to explain this gap might be the diversification of the bioactive substances. Besides the well-known plant secondary products, miRNAs produced by the mother plant may also serve as a mediator between botanicals and health-associated processes. A few studies demonstrated the therapeutics effects of herb-derived miRNAs in mammalian diseases and infections, such as cancer and influenza $[22,24,34,35]$.

Characterization of mammalian target genes of medicinal plant miRNAs using computational and experimental approaches might be a practical way for identifying functional miRNAs. Four features are commonly used for the computational recognition of miRNA targets [72]:

1. Base-pairing between the "seed" (the first 2-8 nucleotides starting at the $5^{\prime}$ end and counting toward the 3 ' end of a mature miRNA) and its target gene;

2. Low free energy (a negative change in overall free energy during a reaction) is expected of the authentic miRNA target pairs;

3. An optional rule for target prediction requires cross-species conservation of the putative binding sites;

4. Site accessibility is an additional rule to measure the ease with which an miRNA can locate and hybridize with an mRNA target.

Using these criterions, it is not surprising to identify the mammalian targets of herbal miRNAs, while approximately 50 human genes were identified as putative targets of MIR168 a [19]. O Table 2 summarizes the currently known miRNAs from medicinal plants (more information such as traditional use/clinical importance and the main bioactive ingredients of these medicinal plants is supplied in Table S1, Supporting Information ), and identification of their potential mammalian targets can be a promising starting point for their cross-kingdom regulatory effects studies. The following up of experimental evidence is required to validate computational predictions since the algorithms based on the physical properties of miRNA regulation may generate many false positive results [73]. On the other hand, bioinformatics tools with additional and more sophisticated analysis allowing more accurate identification of corresponding mRNA targets for these herbal miRNAs need to be developed.

\section{Conclusions and Outlook \\ $\nabla$}

Secondary metabolites serve as bioactive components in medicinal plants, however, the experimental evidence is not convincing regarding their therapeutic effects. It is now documented that the eukaryotic organism of different kingdoms could exchange miRNAs as signals affecting gene expression, indicating the possibility that miRNAs act as a new bioactive component in herbal remedies. The potential of herbal medicine-derived miRNAs in regulating human health or targeting genes associated with diseases are at a very early, exploratory stage. Conclusive validation studies are urgently needed to address this issue. It is necessary to evaluate the stability of herbal miRNAs during the preparation and digestion processes, and in what forms these miRNAs existed. If and to what extent plant-derived miRNAs are absorbed by mammals needs to be investigated. Furthermore, the mechanisms of intestinal absorption, tissue recognition, bioavailability, and function of herbal miRNAs should be established. Given the secondary metabolites presented in herbal preparations, future studies will determine if and how these secondary metabolites influence the stability and uptake of exogenous miRNAs by mammals.

Health regulation by herbal miRNAs is a new concept with a wide range of putative applications, which may include artificial synthesis of therapeutic plant-derived miRNAs that might possess lower side effects, artificially loading miRNAs into plant materials to enhance their in vivo efficacy, and discovery of novel therapeutic target genes. Continuing pursuit in this nascent area will not only broaden our understanding of biological actions of herbal remedies, but also open the ways for the development of new approaches to prevent or treat human diseases. 


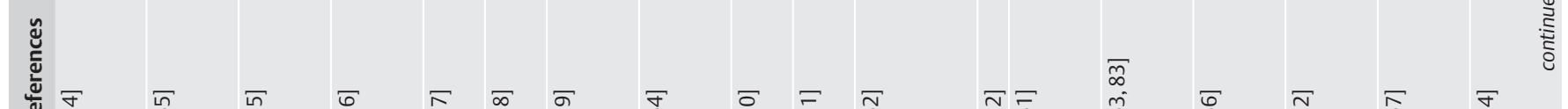

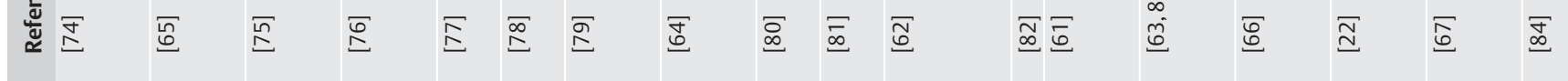

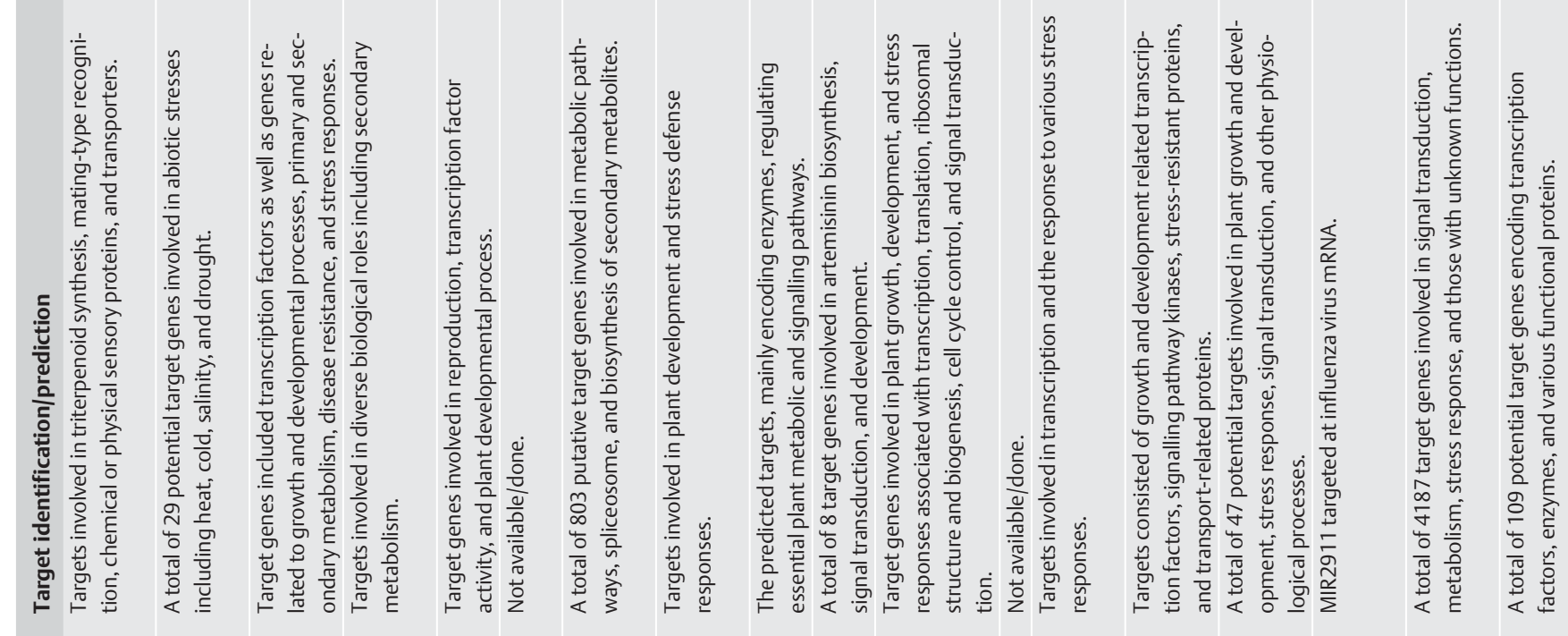

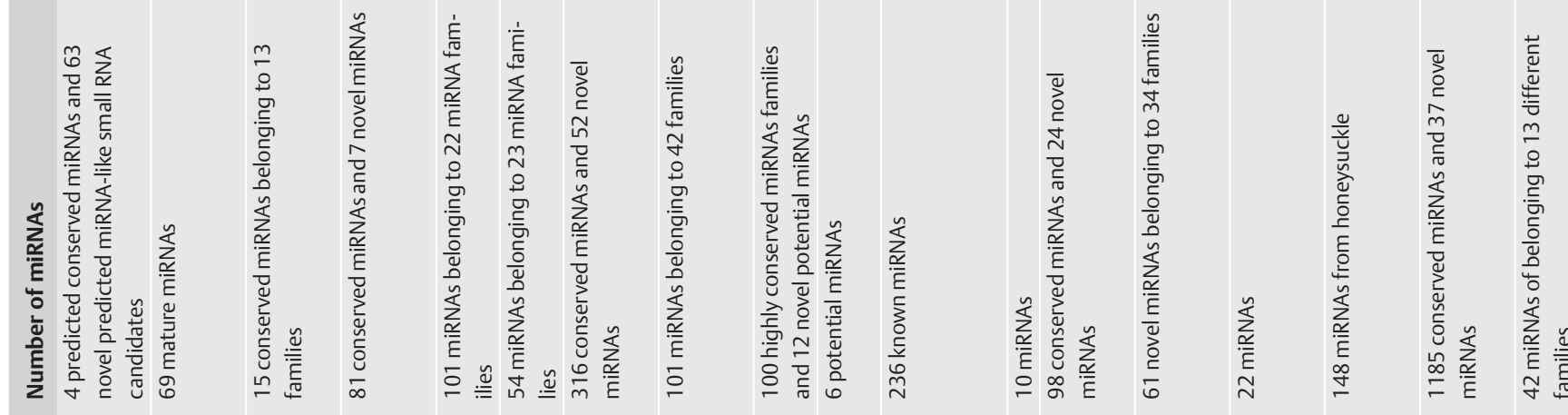

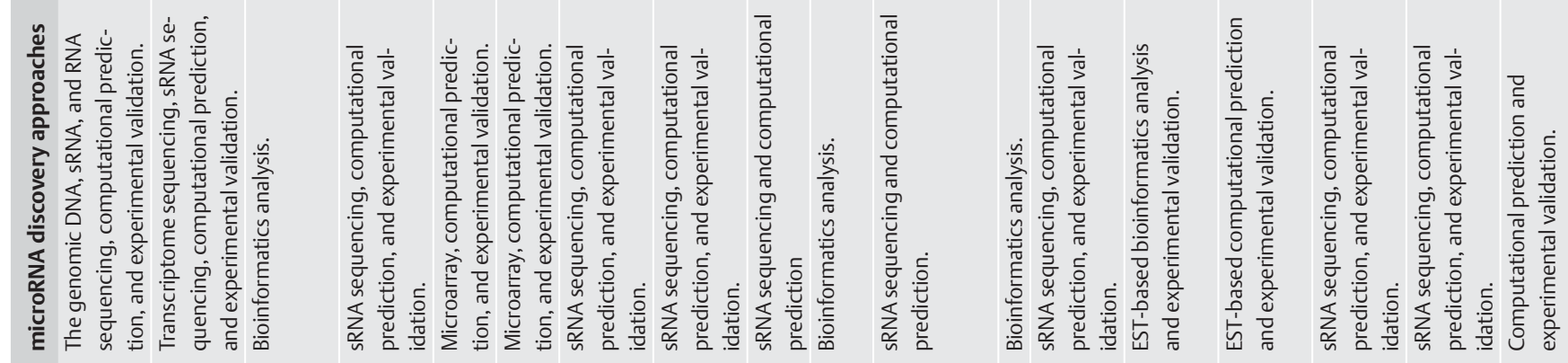

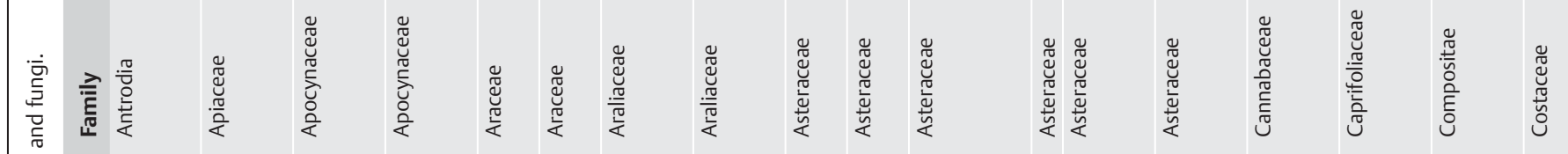

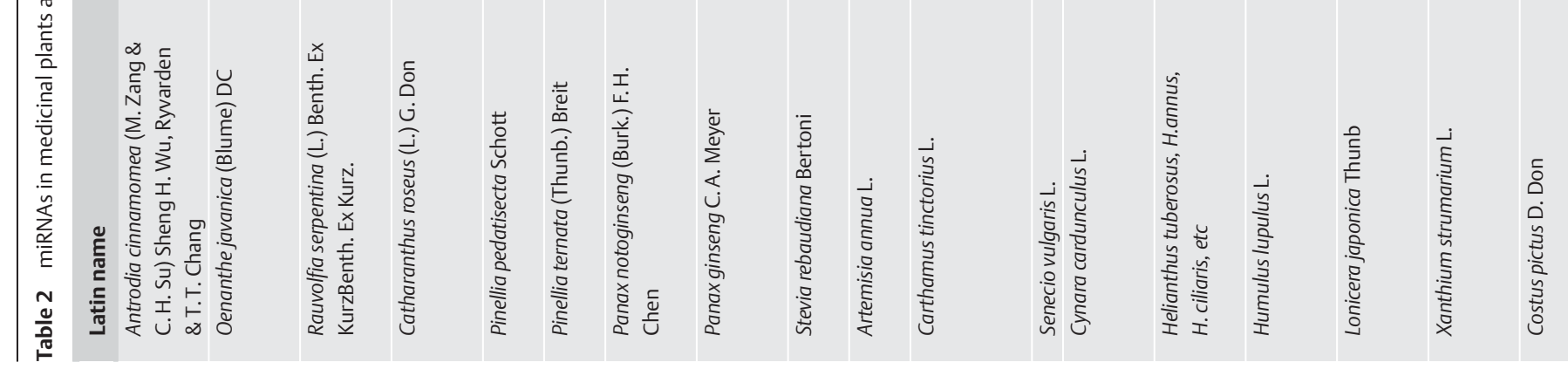


气ัँّำ

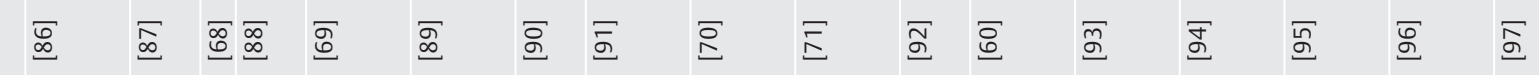

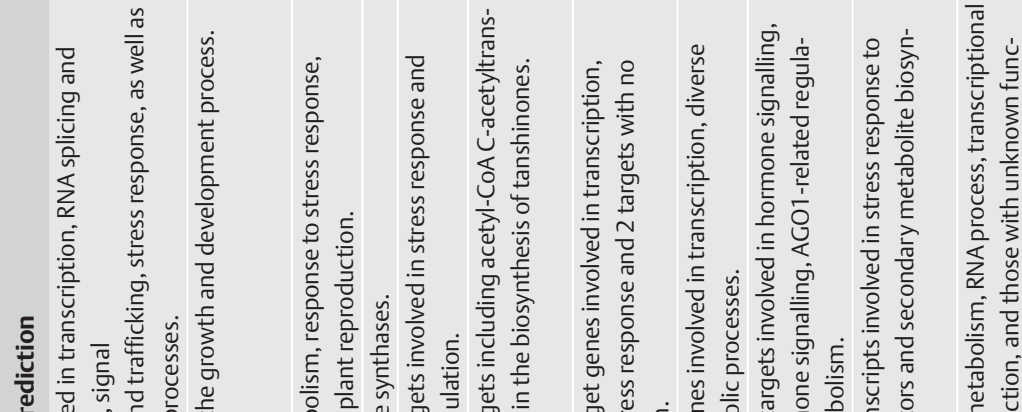

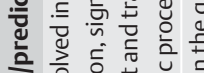

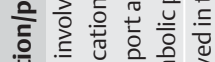

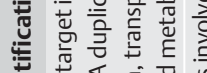

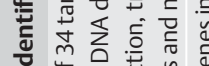

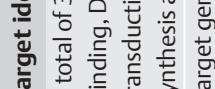

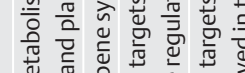

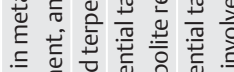

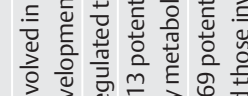

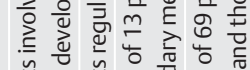

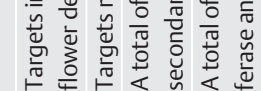

min

MIIIII

II

品皆

Wh

MIII

MIIII

IIIIII)

MII

Int.

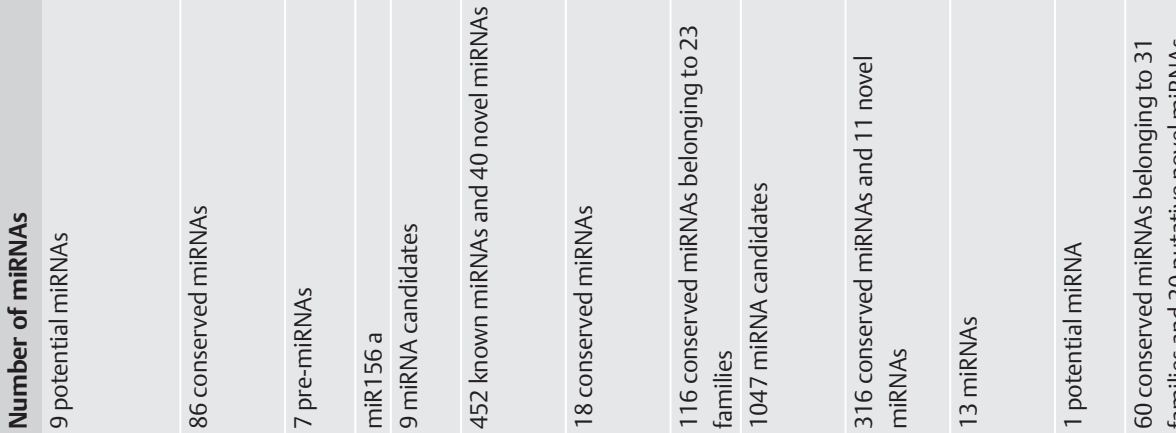
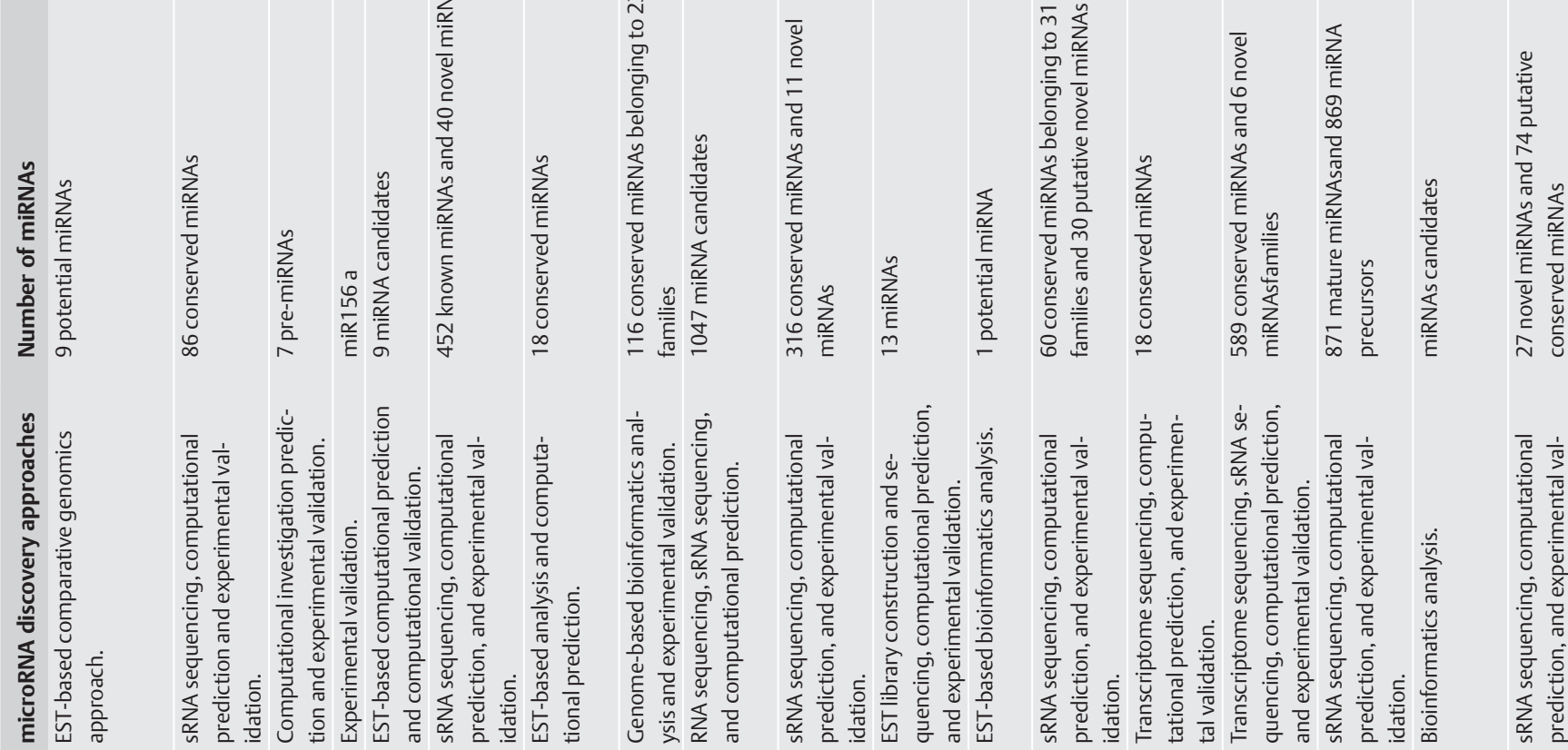

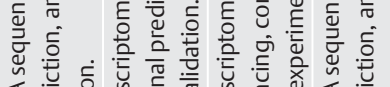

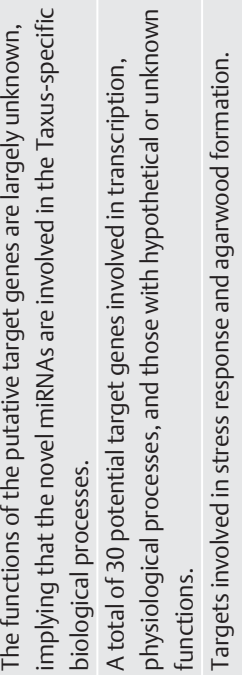

\section{突}

I5

I

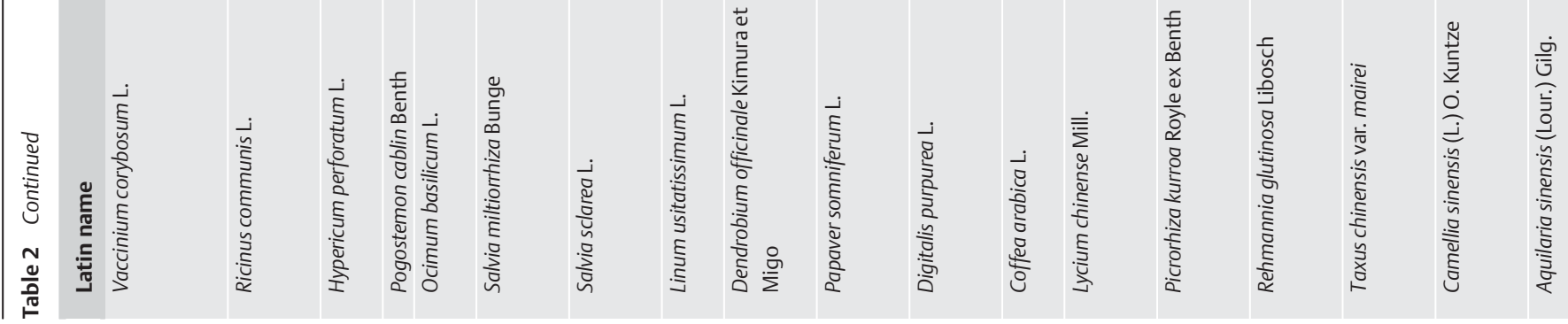




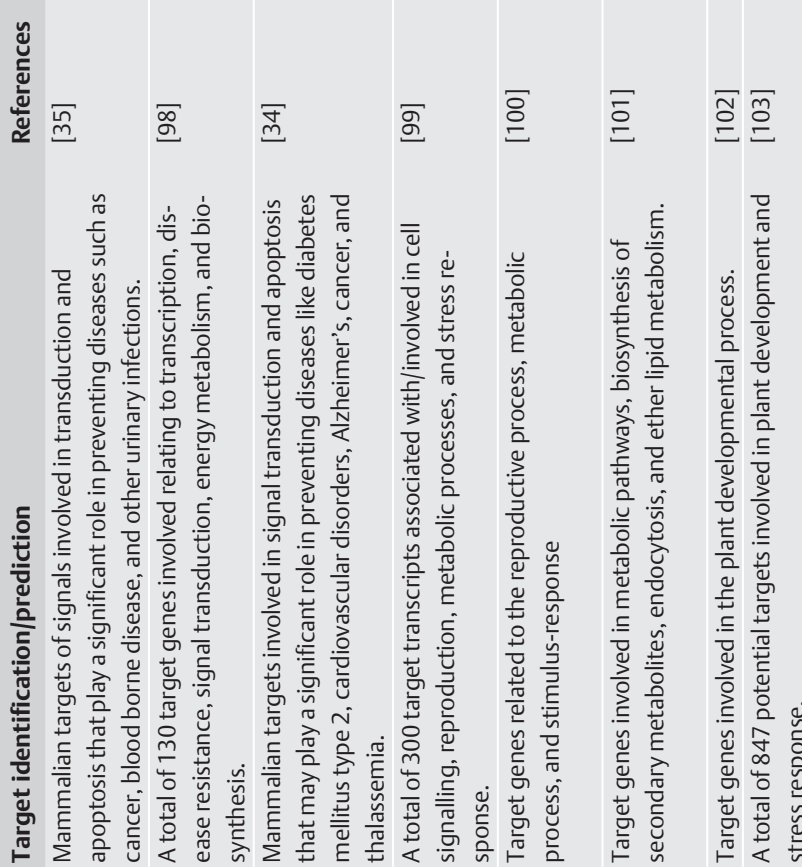

\section{Supporting information}

A table displaying the traditional use or clinical importance and the main bioactive ingredients of medicinal plants with currently known miRNAs is available as Supporting Information.

\section{Acknowledgments}

$\nabla$

This work was financially supported by the FUB - CSC Postdoctoral Research Program (201506300001) of the China Scholarship Council (CSC) and Freie Universität Berlin (FUB).

\section{Conflict of Interest}

$\nabla$

The authors declare that there are no conflicts of interest.

\section{References}

1 Ambros V, Bartel B, Bartel DP, Burge CB, Carrington JC, Chen X, Dreyfuss G, Eddy SR, Griffiths-Jones S, Marshall M, Matzke M, Ruvkun G, Tuschl T. A uniform system for microRNA annotation. RNA 2003; 9: 277-279

2 Ling H, Fabbri M, Calin GA. MicroRNAs and other non-coding RNAs as targets for anticancer drug development. Nat Rev Drug Discov 2013; 12: 847-865

3 Friedman RC, Farh KKH, Burge CB, Bartel DP. Most mammalian mRNAs are conserved targets of microRNAs. Genome Res 2009; 19: 92-105

4 Ranganathan K, Sivasankar V. MicroRNAs - biology and clinical applications. J Oral Maxillofac Pathol 2014; 18: 229-234

5 Van Rooij E. Introduction to the series on microRNAs in the cardiovascular system. Circ Res 2012; 110: 481-482

6 Rottiers V, Näär AM. MicroRNAs in metabolism and metabolic disorders. Nat Rev Mol Cell Biol 2012; 13: 239-250

7 Salta E, De Strooper B. Non-coding RNAs with essential roles in neurodegenerative disorders. Lancet Neurol 2012; 11: 189-200

8 Rothschild SI. MicroRNA therapies in cancer. Mol Cell Ther 2014; 2: 7

9 Trang P, Wiggins JF, Daige CL, Cho C, Omotola M, Brown D, Weidhaas JB, Bader AG, Slack FJ. Systemic delivery of tumor suppressor microRNA mimics using a neutral lipid emulsion inhibits lung tumors in mice. Mol Ther 2011; 19: 1116-1122

10 Garzon R, Heaphy CEA, Havelange V, Fabbri M, Volinia S, Tsao T, Zanesi N, Kornblau SM, Marcucci G, Calin GA, Andreeff M, Croce CM. MicroRNA 29b functions in acute myeloid leukemia. Blood 2009; 114: 5331-5341

11 Cubillos-Ruiz JR, Baird JR, Tesone AJ, Rutkowski MR, Scarlett UK, Camposeco-Jacobs AL, Anadon-Arnillas J, Harwood NM, Korc M, Fiering $S N$, Sempere LF, Conejo-Garcia JR. Reprogramming tumor-associated dendritic cells in vivo using miRNA mimetics triggers protective immunity against ovarian cancer. Cancer Res 2012; 72: 1683-1693

12 Bouchie A. First microRNA mimic enters clinic. Nat Biotechnol 2013; 31: 577

13 Li Z, Rana TM. Therapeutic targeting of microRNAs: current status and future challenges. Nat Rev Drug Discov 2014; 13: 622-638

14 Van Rooij E, Kauppinen S. Development of microRNA therapeutics is coming of age. EMBO Mol Med 2014; 6: 851-864

15 Zhang Z, Song C, Zhang F, Xiang L, Chen Y, Li Y, Pan J, Liu H, Xiao GG, Ju D. Rhizoma Dioscoreae extract protects against alveolar bone loss in ovariectomized rats via microRNAs regulation. Nutrients 2015; 7: $1333-1351$

16 Wu N, Wu G, Hu R, Li M, Feng H. Ginsenoside Rh2 inhibits glioma cell proliferation by targeting microRNA-128. Acta Pharmacol Sin 2011; 32: 345-353

17 Hu H, Li K, Wang X, Liu Y, Lu Z, Dong R, Guo H, Zhang M. Set9, NF-kB, and microRNA-21 mediate berberine-induced apoptosis of human multiple myeloma cells. Acta Pharmacol Sin 2013; 34: 157-166

18 Zhang Y, Zhang GB, Xu XM, Zhang M, Qu D, Niu HY, Bai X, Kan L, He P. Suppression of growth of A549 lung cancer cells by waltonitone and its mechanisms of action. Oncol Rep 2012; 28: 1029-1035

19 Zhang L, Hou D, Chen X, Li D, Zhu L, Zhang Y, Li J, Bian Z, Liang X, Cai X, Yin $Y$, Wang C, Zhang T, Zhu D, Zhang D, Xu J, Chen $Q$ Ba Y, Liu J, Wang $Q$ Chen J, Wang J, Wang M, Zhang $Q$ Z Zhang J, Zen K, Zhang CY. Exogenous plant MIR168 a specifically targets mammalian LDLRAP1: evidence of cross-kingdom regulation by microRNA. Cell Res 2012; 22: 273-274 
20 Yang J, Farmer LM, Agyekum AA, Hirschi KD. Detection of dietary plantbased small RNAs in animals. Cell Res 2015; 25: 517-520

21 Liang H, Zhang S, Fu Z, Wang Y, Wang N, Liu Y, Zhao C, Wu J, Hu Y, Zhang $J$, Chen X, Zen K, Zhang CY. Effective detection and quantification of dietetically absorbed plant microRNAs in human plasma. J Nutr Biochem 2015; 26: 505-512

22 Zhou Z, Li X, Liu J, Dong L, Chen Q Liu J, Kong H, Zhang Q, Qi X, Hou D, Zhang L, Zhang G, Liu Y, Zhang Y, Li J, Wang J, Chen X, Wang H, Zhang J, Chen $H$, Zen $K$, Zhang $C Y$. Honeysuckle-encoded atypical microRNA2911 directly targets influenza A viruses. Cell Res 2014; 25 : 39-49

23 Mlotshwa S, Pruss GJ, MacArthur JL, Endres MW, Davis C, Hofseth LJ, Peña $M M$, Vance $V$. A novel chemopreventive strategy based on therapeutic microRNAs produced in plants. Cell Res 2015; 25: 521-524

24 Chin AR, Fong MY, Somlo G, Wu J, Swiderski P, Wu X, Wang SE. Crosskingdom inhibition of breast cancer growth by plant miR159. Cell Res 2016; 26: 217-228

25 Wang K, Li H, Yuan Y, Etheridge A, Zhou Y, Huang D, Wilmes P, Galas D. The complex exogenous RNA spectra in human plasma: an interface with human gut biota? PLoS One 2012; 7: e51009

26 Liang G, Zhu Y, Sun B, Shao Y, Jing A, Wang J, Xiao Z. Assessing the survival of exogenous plant microRNA in mice. Food Sci Nutr 2014; 2 : 380-388

27 Lukasik A, Zielenkiewicz P. In silico identification of plant miRNAs in mammalian breast milk exosomes - a small step forward? PLoS One 2014; 9: e99963

28 Snow JW, Hale AE, Isaacs SK, Baggish AL, Chan SY. Ineffective delivery of diet-derived microRNAs to recipient animal organisms. RNA Biol 2013; 10: 1107-1116

29 Witwer KW, McAlexander MA, Queen SE, Adams RJ. Real-time quantitative PCR and droplet digital PCR for plant miRNAs in mammalian blood provide little evidence for general uptake of dietary miRNAs: limited evidence for general uptake of dietary plant xenomiRs. RNA Biol 2013; 10: 1080-1086

30 Dickinson B, Zhang Y, PetrickJS, Heck G, Ivashuta S, Marshall WS. Lack of detectable oral bioavailability of plant microRNAs after feeding in mice. Nat Biotechnol 2013; 31: 967-969

31 Zhang Y, Wiggins BE, Lawrence C, Petrick J, Ivashuta S, Heck G. Analysis of plant-derived miRNAs in animal small RNA datasets. BMC Genomics 2012; 13: 381

32 Masood M, Everett CP, Chan SY, Snow JW. Negligible uptake and transfer of diet-derived pollen microRNAs in adult honey bees. RNA Biol 2016 13: 109-118

33 Yang J, Farmer LM, Agyekum AAA, Elbaz-Younes I, Hirschi KD. Detection of an abundant plant-based small RNA in healthy consumers. PLoS One 2015; 10: e0137516

34 Rameshwari R, Singhal D, Narang R, Maheshwari A, Prasad TV. In silico prediction of miRNA in Curcuma longa and their role in human metabolomics. Int J Biotechnol Res 2013; 4: 253-259

35 Dubey A, Kalra SS, Trivedi N. Computational prediction of miRNA in Gmelina arborea and their role in human metabolomics. Am J Biosci Bioeng 2013; 1: 62-74

36 Chen X, Liang H, Zhang J, Zen K, Zhang CY. Secreted microRNAs: a new form of intercellular communication. Trends Cell Biol 2012; 22: 125132

37 Ju S, Mu J, Dokland T, Zhuang X, Wang Q Jiang H, Xiang X, Deng ZB, Wang $B$, Zhang L, Roth M, Welti R, Mobley J, Jun Y, Miller D, Zhang HG. Grape exosome-like nanoparticles induce intestinal stem cells and protect mice from DSS-induced colitis. Mol Ther 2013; 21: 1345-1357

38 Mu J, Zhuang X, Wang Q Jiang H, Deng ZB, Wang B, Zhang L, Kakar S, Jun $Y$, Miller D, Zhang HG. Interspecies communication between plant and mouse gut host cells through edible plant derived exosome-like nanoparticles. Mol Nutr Food Res 2014; 58: 1561-1573

39 Philip A, Ferro VA, Tate RJ. Determination of the potential bioavailability of plant microRNAs using a simulated human digestion process. Mol Nutr Food Res 2015; 59: 1962-1972

40 Li J, Yang Z, Yu B, Liu J, Chen X. Methylation protects miRNAs and siRNAs from a 3'-end uridylation activity in Arabidopsis. Curr Biol 2005; 15 : 1501-1507

41 Voinnet 0 . Origin, biogenesis, and activity of plant microRNAs. Cell 2009; 136: 669-687

42 Winter J, Diederichs S. Argonaute proteins regulate microRNA stability: Increased microRNA abundance by Argonaute proteins is due to microRNA stabilization. RNA Biol 2011; 8: 1149-1157
43 Nolte-'t Hoen EN, Van Rooij E, Bushell M, Zhang CY, Dashwood RH, James WPT, Harris C, Baltimore D. The role of microRNA in nutritional control. J Intern Med 2015; 278: 99-109

44 Zhao Y, Mo B, Chen X. Mechanisms that impact microRNA stability in plants. RNA Biol 2012; 9: 1218-1223

45 Wang K, Zhang S, Weber J, Baxter D, Galas DJ. Export of microRNAs and microRNA-protective protein by mammalian cells. Nucleic Acids Res 2010; 38: 7248-7259

46 Köberle V, Pleli T, Schmithals C, Augusto Alonso E, Haupenthal J, Bönig H, Peveling-Oberhag J, Biondi RM, Zeuzem S, Kronenberger B, Waidmann $O$, Piiper A. Differential stability of cell-free circulating microRNAs: implications for their utilization as biomarkers. PLoS One 2013; 8: e75184

47 Beltrami C, Clayton A, Newbury L, Corish P, Jenkins R, Phillips A, Fraser D, Bowen $T$. Stabilization of urinary microRNAs by association with exosomes and argonaute 2 protein. Non-Coding RNA 2015; 1: 151-166

48 Dutta S, Basak A, Dasgupta S. Synthesis and ribonuclease A inhibition activity of resorcinol and phloroglucinol derivatives of catechin and epicatechin: Importance of hydroxyl groups. Bioorg Med Chem 2010; 18: 6538-6546

49 Ghosh KS, Maiti TK, Debnath J, Dasgupta S. Inhibition of Ribonuclease A by polyphenols present in green tea. Proteins 2007; 69: 566-580

50 Tai BH, Nhut ND, Nhiem NX, Tung NH, Quang TH, Thuy Luyen BT, Huong TT, Wilson J, Beutler JA, Ban NK, Cuong NM, Kim YH. Evaluation of the RNase $\mathrm{H}$ inhibitory properties of Vietnamese medicinal plant extracts and natural compounds. Pharm Biol 2011; 49: 1046-1051

51 Dutta S, Bhattacharyya D. Enzymatic, antimicrobial and toxicity studies of the aqueous extract of Ananas comosus (pineapple) crown leaf. J Ethnopharmacol 2013; 150: 451-457

52 Witwer KW. XenomiRs and miRNA homeostasis in health and disease: evidence that diet and dietary miRNAs directly and indirectly influence circulating miRNA profiles. RNA Biol 2012; 9: 1147-1154

53 Witwer KW, Hirschi KD. Transfer and functional consequences of dietary microRNAs in vertebrates: concepts in search of corroboration: negative results challenge the hypothesis that dietary xenomiRs cross the gut and regulate genes in ingesting vertebrates, but important questions persist. Bioessays 2014; 36: 394-406

54 Aouadi M, Tesz GJ, Nicoloro SM, Wang M, Chouinard M, Soto E, Ostroff GR, Czech MP. Orally delivered siRNA targeting macrophage Map4k4 suppresses systemic inflammation. Nature 2009; 458: 1180-1184

55 Robbins PD, Morelli AE. Regulation of immune responses by extracellular vesicles. Nat Rev Immunol 2014; 14: 195-208

56 Fabbri M. TLRs as miRNA receptors. Cancer Res 2012; 72: 6333-6337

57 Mulcahy LA, Pink RC, Carter DRF. Routes and mechanisms of extracellular vesicle uptake. J Extracell Vesicles 2014; 3: 24641

58 Li C, Zhu Y, Guo X, Sun C, Luo H, Song J, Li Y, Wang L, Qian J, Chen S. Transcriptome analysis reveals ginsenosides biosynthetic genes, microRNAs and simple sequence repeats in Panax ginseng C. A. Meyer BMC Genomics 2013; 14: 245

59 Zhang $L, W u$ B, Zhao D, Li C, Shao F, Lu S. Genome-wide analysis and molecular dissection of the SPL gene family in Salvia miltiorrhiza. J Integr Plant Biol 2014; 56: 38-50

60 Khaldun ABM, Huang W, Liao S, Lv H, Wang Y. Identification of microRNAs and target genes in the fruit and shoot tip of Lycium chinense: a traditional Chinese medicinal plant. PLoS One 2015; 10: e0116334

61 De Paola D, Cattonaro F, Pignone D, Sonnante G. The miRNAome of globe artichoke: conserved and novel micro RNAs and target analysis. BMC Genomics 2012; 13: 41

62 Li H, Dong Y, Sun Y, Zhu E, Yang J, Liu X, Xue P, Xiao Y, Yang S, Wu J, Li X Investigation of the microRNAs in safflower seed, leaf, and petal by high-throughput sequencing. Planta 2011; 233: 611-619

63 Barozai MYK, Baloch IA, Din M. Identification of MicroRNAs and their targets in Helianthus. Mol Biol Rep 2012; 39: 2523-2532

64 Wu B. Wang M, Ma Y, Yuan L, Lu S. High-throughput sequencing and characterization of the small RNA transcriptome reveal features of novel and conserved microRNAs in Panax ginseng. PLoS One 2012; 7: e44385

65 Jiang $Q$, Wang F, Tan HW, Li MY, Xu ZS, Tan GF, Xiong AS. De novo transcriptome assembly, gene annotation, marker development, and miRNA potential target genes validation under abiotic stresses in Oenanthe javanica. Mol Genet Genomics 2015; 290: 671-683

66 Mishra AK, Duraisamy GS, Týcová A, Matoušek J. Computational exploration of microRNAs from expressed sequence tags of Humulus lupulus, target predictions and expression analysis. Comput Biol Chem 2015; 59: $131-141$ 
67 Fan R, Li Y, Li C, Zhang Y. Differential microRNA analysis of glandular trichomes and young leaves in Xanthium strumarium L. reveals their putative roles in regulating terpenoid biosynthesis. PLoS One 2015; 10: e0139002

68 Yu ZX, Wang LJ, Zhao B, Shan CM, Zhang YH, Chen DF, Chen XY. Progressive regulation of sesquiterpene biosynthesis in Arabidopsis and Patchouli (Pogostemon cablin) by the miR156-targeted SPL transcription factors. Mol Plant 2015; 8: 98-110

69 Xu X, Jiang $Q$ Ma X, Ying $Q$ Shen B, Qian Y, Song H, Wang H. Deep sequencing identifies tissue-specific microRNAs and their target genes involving in the biosynthesis of tanshinones in Salvia miltiorrhiza. PLoS One 2014; 9: e111679

70 Boke H, Ozhuner E, Turktas M, Parmaksiz I, Ozcan S, Unver T. Regulation of the alkaloid biosynthesis by miRNA in opium poppy. Plant Biotechnol J 2015; 13: 409-420

71 Wu B, Li Y, Yan H, Ma Y, Luo H, Yuan L, Chen S, Lu S. Comprehensive transcriptome analysis reveals novel genes involved in cardiac glycoside biosynthesis and mlncRNAs associated with secondary metabolism and stress response in Digitalis purpurea. BMC Genomics 2012; 13: 15

72 Peterson SM, Thompson JA, Ufkin ML, Sathyanarayana P, Liaw L, Congdon CB. Common features of microRNA target prediction tools. Front Genet 2014; $5: 23$

73 Ekimler S, Sahin K. Computational methods for microRNA target prediction. Genes (Basel) 2014; 5: 671-683

74 Lin YL, Ma LT, Lee YR, Lin SS, Wang SY, Chang TT, Shaw JF, Li WH, Chu FH. MicroRNA-like small RNAs prediction in the development of Antrodia cinnamomea. PLoS One 2015; 10: e0123245

75 Prakash P, Rajakani R, Gupta V. Transcriptome-wide identification of Rauvolfia serpentina microRNAs and prediction of their potential targets. Comput Biol Chem 2015; 61: 62-74

76 Prakash P, Ghosliya D, Gupta V. Identification of conserved and novel microRNAs in Catharanthus roseus by deep sequencing and computational prediction of their potential targets. Gene 2015; 554: 181-195

77 Dong $M$, Yang $D$, Lang $Q$ Zhou $W, X u S$, Xu T. Microarray and degradome sequencing reveal microRNA differential expression profiles and their targets in Pinellia pedatisecta. PLoS One 2013; 8: e75978

78 Wang B, Dong M, Chen W, Liu X, Feng R, Xu T. Microarray identification of conserved microRNAs in Pinellia pedatisecta. Gene 2012; 498: 3640

79 Wei R, Qiu D, Wilson IW, Zhao H, Lu S, Miao J, Feng S, Bai L, Wu Q, Tu D, Ma X, Tang $Q$. Identification of novel and conserved microRNAs in Panax notoginseng roots by high-throughput sequencing. BMC Genomics 2015; 16: 835

80 Mandhan V, Kaur J, Singh K. smRNAome profiling to identify conserved and novel microRNAs in Stevia rebaudiana Bertoni. BMC Plant Biol 2012; 12: 197

81 Pani A, Mahapatra RK, Behera N, Naik PK. Computational identification of sweet wormwood (Artemisia annua) microRNA and their mRNA targets. Genomics Proteomics Bioinformatics 2011; 9: 200-210

82 Sahu S, Khushwaha A, Dixit R. Computational identification of miRNAs in medicinal plant Senecio vulgaris (Groundsel). Bioinformation 2011; 7: $375-378$

83 Johansson E, Prade T, Angelidaki I, Svensson SE, Newson WR, Gunnarsson $I B$, Hovmalm HP. Economically viable components from Jerusalem artichoke (Helianthus tuberosus L.) in a biorefinery concept. Int J Mol Sci 2015; 16: 8997-9016

84 Das A, Das P, Kalita MC, Mondal TK. Computational identification, target prediction, and validation of conserved miRNAs in insulin plant (Costus pictus D. Don). Appl Biochem Biotechnol 2016; 178: 513-526

85 Li X, Hou Y, Zhang L, Zhang W, Quan C, Cui Y, Bian S. Computational identification of conserved microRNAs and their targets from expression sequence tags of blueberry (Vaccinium corybosum). Plant Signal Behav 2014; 9: e29462

86 Xu W, Cui Q Li F, Liu A. Transcriptome-wide identification and characterization of microRNAs from castor bean (Ricinus communis L.). PLoS One 2013; 8: e69995
87 Galla G, Volpato M, Sharbel TF, Barcaccia G. Computational identification of conserved microRNAs and their putative targets in the Hypericum perforatum L. flower transcriptome. Plant Reprod 2013; 26: $209-229$

88 Singh N, Sharma A. In-silico identification of miRNAs and their regulating target functions in Ocimum basilicum. Gene 2014; 552: 277282

89 Legrand S, Valot N, Nicolé F, Moja S, Baudino S, Jullien F, Magnard JL, Caissard JC, Legendre L. One-step identification of conserved miRNAs, their targets, potential transcription factors and effector genes of complete secondary metabolism pathways after 454 pyrosequencing of calyx cDNAs from the labiate Salvia sclarea L. Gene 2010; 450: 5562

90 Barvkar VT, Pardeshi VC, Kale SM, Qiu S, Rollins M, Datla R, Gupta VS, Kadoo NY. Genome-wide identification and characterization of microRNA genes and their targets in flax (Linum usitatissimum): Characterization of flax miRNA genes. Planta 2013; 237: 1149-1161

91 Meng Y, Yu D, Xue J, Lu J, Feng S, Shen C, Wang H. A transcriptome-wide, organ-specific regulatory map of Dendrobium officinale, an important traditional Chinese orchid herb. Sci Rep 2016; 6: 18864

92 Akter A, Islam MM, Mondal SI, Mahmud Z, Jewel NA, Ferdous S, Amin MR, Rahman MM. Computational identification of miRNA and targets from expressed sequence tags of coffee (Coffea arabica). Saudi J Biol Sci 2014; 21: 3-12

93 Vashisht I, Mishra P, Pal T, Chanumolu S, Singh TR, Chauhan RS. Mining NGS transcriptomes for miRNAs and dissecting their role in regulating growth, development, and secondary metabolites production in different organs of a medicinal herb, Picrorhiza kurroa. Planta 2015; 241: 1255-1268

94 Yang Y, Chen X, Chen J, Xu H, Li J, Zhang Z. Differential miRNA expression in Rehmannia glutinosa plants subjected to continuous cropping. BMC Plant Biol 2011; 11: 53

95 Hao DC, Yang L, Xiao PG, Liu M. Identification of Taxus microRNAs and their targets with high-throughput sequencing and degradome analysis. Physiol Plant 2012; 146: 388-403

96 Prabu GR, Mandal AKA. Computational identification of miRNAs and their target genes from expressed sequence tags of tea (Camellia sinensis). Genomics Proteomics Bioinformatics 2010; 8: 113-121

97 Gao ZH, Wei JH, Yang Y, Zhang Z, Xiong HY, Zhao WT. Identification of conserved and novel microRNAs in Aquilaria sinensis based on small RNA sequencing and transcriptome sequence data. Gene 2012; 505: 167-175

98 Wang C, Wang X, Kibet NK, Song C, Zhang C, Li X, Han J, Fang J. Deep sequencing of grapevine flower and berry short RNA library for discovery of novel microRNAs and validation of precise sequences of grapevine microRNAs deposited in miRBase. Physiol Plant 2011; 143: 64-81

99 Singh N, Srivastava S, Sharma A. Identification and analysis of miRNAs and their targets in ginger using bioinformatics approach. Gene 2016; 575: 570-576

100 Zhang $Q$ Li J, Sang Y, Xing S, Wu Q Liu X. Identification and characterization of microRNAs in Ginkgo biloba var. epiphylla Mak. PLoS One 2015; 10: e0127184

101 Zhao D, Gong S, Hao Z, Tao J. Identification of miRNAs responsive to Botrytis cinerea in herbaceous peony (Paeonia lactiflora Pall.) by high-throughput sequencing. Genes (Basel) 2015; 6: 918-934

102 Thirugnanasambantham K, Saravanan S, Karikalan K, Bharanidharan $R$, Lalitha P, Ilango S, Hairullslam VI. Identification of evolutionarily conserved Momordica charantia microRNAs using computational approach and its utility in phylogeny analysis. Comput Biol Chem 2015; 58: 25-39

103 Pan L, Wang X, Jin J, Yu X, Hu J. Bioinformatic identification and expression analysis of Nelumbo nucifera microRNA and their targets. Appl Plant Sci 2015; 3: 1500046 